\title{
Olaparib maintenance for first-line treatment of ovarian cancer- Will SOLO1 reset the standard of care?
}

\begin{abstract}
Maintenance therapy with PARP inhibitors has heralded a new era in the management of recurrent epithelial ovarian cancer. The greatest effect is seen in women with $B R C A 1 / 2$ tumours but those without this mutation also benefit. However, in most patients, the drugs eventually fail to prevent progression, so alternative strategies are needed. The SOLO1 trial randomised women with BRCA1/2 mutated advanced ovarian cancer to olaparib or placebo maintenance after first-line chemotherapy. Olaparib significantly improved progression-free survival to a degree that has not been seen in other first-line trials in ovarian cancer. This landmark trial is likely to change practice for this group of women. Here we focus on the SOLO1 results in the context of the current management of advanced ovarian cancer.
\end{abstract}

Key Words: ovarian cancer, PARP inhibitor, olaparib, SOLO1, BRCA mutation

\section{Introduction}

Most women with newly diagnosed advanced ovarian, primary peritoneal or fallopian-tube cancer (subsequently referred to as ovarian cancer) will relapse within three years after standard treatment with surgery and platinum-based chemotherapy [1]. The benefit of oral poly adenosine diphosphate-ribose polymerase (PARP) inhibitors as maintenance therapy in relapsed disease has been well established [26]. Olaparib, niraparib and rucaparib all delay progression following platinum-based chemotherapy in high grade ovarian cancer with benefit seen in all groups of patients, but the greatest benefit is observed in those with a $B R C A 1$ or $B R C A 2$ (BRCA1/2) mutation, either germline or somatic [2-5]. The median progression free survival (PFS) from randomisation ranges from 11.2-21 months for $B R C A 1 / 2$ mutant populations and 7.4-10.8 months for BRCA1/2 wild-type/all comer populations [2-6]. To date, no benefit has been observed in overall survival (OS), but with long-term follow up in Study $19,11 \%$ of patients remained on olaparib for over six years without evidence of tumour progression [7]. Despite impressive prolongation in PFS, disease relapse remains almost inevitable, even within the BRCA1/2 mutant population. The consistent clinical benefit seen using different PARP inhibitors in recurrent ovarian cancer raises the question whether the strategy of maintenance therapy post chemotherapy could be applied to the first-line setting. The SOLO1 trial with olaparib is the first study to evaluate PARP inhibitor maintenance therapy in this setting, to determine whether a greater benefit can be derived with the early introduction of PARP inhibition in the BRCA1/2 mutant population [8].

\section{SOLO1 Trial design and summary of result and toxicity}


SOLO1 is an international, randomised, double-blind, phase 3 trial conducted in 391 patients with newly diagnosed advanced high-grade serous or endometrioid ovarian, primary peritoneal or fallopian-tube cancer and with a mutation in BRCA1, BRCA2, or both (Figure 1) [8]. Eligible patients were diagnosed with FIGO stage III or IV disease and had a complete or partial clinical response after platinum-based chemotherapy. Patients with stage III disease had undergone an attempt at cytoreductive surgery before commencing chemotherapy (up front) or after the start but before the end of chemotherapy (interval debulking surgery), and patients with stage IV disease had undergone either biopsy or up front or interval cytoreductive surgery. Eligible patients had a deleterious or suspected deleterious germline or somatic $B R C A 1 / 2$ mutation, as determined by local or central testing.

391 patients were randomly assigned, in a 2:1 ratio, to receive olaparib tablets (300 mg twice daily, $\mathrm{n}=$ $260)$ or placebo ( $n=131)$. Trial intervention was continued until investigator-assessed disease progression. Patients who had no evidence of disease at two years discontinued treatment, but patients who had a partial response at two years were permitted to continue receiving the treatment. Crossover between trial groups was not permitted. The primary end point was investigator assessed progression-free survival. Enrolment was allowed up to eight weeks following the completion of chemotherapy.

The trial was considered positive after a median follow-up of 41 months; all patients had been followed for at least 36 months. The risk of investigator assessed disease progression or death (data maturity $51 \%$ ) was $70 \%$ lower with olaparib than with placebo (60\% versus $27 \%$; hazard ratio $(\mathrm{HR}) 0.30 ; 95 \% \mathrm{Cl}, 0.23$ to $0.41 ; \mathrm{p}<0.001$ ). In the analysis of PFS (as assessed by blinded independent central review (BICR), data maturity, 38\%), the Kaplan-Meier estimate of the rate of freedom from disease progression and death at 3 years was $69 \%$ in the olaparib group, as compared with $35 \%$ in the placebo group ( $\mathrm{HR} 0.28 ; 95 \% \mathrm{Cl}, 0.20$ to $0.39 ; p<0.001$ ) [8]. These results are consistent with the benefit of olaparib with regard to progressionfree survival as assessed by investigators [8].

Adverse events were consistent with the known toxic effects of olaparib seen in similar patients undergoing maintenance treatment for recurrent ovarian cancer [3,6]. $98 \%$ and $92 \%$ of patient receiving olaparib and placebo respectively experienced at least one adverse events with the most common being nausea $(77 \%$ olaparib versus $38 \%$ placebo), fatigue/asthenia ( $63 \%$ olaparib versus $42 \%$ placebo), vomiting ( $40 \%$ olaparib versus $15 \%$ placebo) and anaemia (39\% olaparib versus $10 \%$ placebo). Serious adverse events occurred in $21 \%$ of olaparib treated patients and $12 \%$ of those receiving placebo, with anaemia the most common ( $7 \%$ olaparib versus $0 \%$ placebo). There were no treatment related deaths. Adverse events resulted in dose reduction and treatment discontinuation in $28 \%$ and $12 \%$ of olaparib treated patients and $3 \%$ and $2 \%$ of those on placebo respectively. Neither trial group had a clinically significant change in health-related quality of life [8].

\section{BRCA1/2 mutations and PARP inhibition}


Inherited mutations in $B R C A 1$ and $B R C A 2$ genes are responsible for the majority of familial ovarian cancer syndromes. BRCA1 and BRCA2 are essential components of the highly conserved homologousrecombination repair pathway, which is responsible for the error-free repair of DNA double-strand breaks and therefore critical for maintaining genomic stability [9]. Germline mutations in BRCA1/2 account for approximately $13-15 \%$ of all high grade ovarian cancers $[10,11]$ with an additional $5-7 \%$ of patients harbouring somatic $B R C A 1 / 2$ mutations [10,11]. The finding that single-agent PARP inhibition selectively killed $B R C A 1 / 2$ deficient cells was a key discovery in exploiting synthetic lethal approaches in oncology $[12,13]$. PARP inhibitors trap PARP protein onto DNA at sites of single-strand DNA breaks. When this trapped PARP is encountered by the DNA replication machinery it leads to stalling of the replication fork, collapse and the generation of a double strand break, which cannot be repaired in cells with defective homologous recombination, such as $B R C A 1 / 2$ mutated cells [14]. In addition to the familial ovarian cancer syndromes, a number of sporadic ovarian cancers are also defective in homologous-recombination repair and share the deficient phenotype associated with $B R C A 1 / 2[15,16]$. Initial indications for PARP inhibition were limited to tumours characterized by either a germline or somatic $B R C A 1 / 2$ mutation, with olaparib receiving the first license from the EMA as maintenance treatment of recurrent $B R C A 1 / 2$ mutated epithelial ovarian cancer [17]. Emerging data, supports an extended scope for PARP inhibitor use and this has been reflected with the recent approval by the FDA and EMA for, niraparib, olaparib tablets and rucaparib as maintenance therapy for all patients, treated with two or more prior chemotherapy regimens who have responded to platinum therapy, regardless of $B R C A 1 / 2$ status [17-22]. Olaparib and rucaparib have additional licences as monotherapy for recurrent $B R C A 1 / 2$ mutant ovarian cancer $[17,19,21,22]$. Response rates of between $31 \%$ and $41 \%$ in $B R C A 1 / 2$ mutation carriers and up to $24 \%$ in $B R C A$ wild-type patients have been demonstrated with olaparib monotherapy [23,24]. Similarly, within Study 10 and ARIEL2 trials, there was an overall response rate of $53.8 \%(95 \% \mathrm{Cl} 43.8-63.5)$ with rucaparib monotherapy in $B R C A 1 / 2$ mutated patients following at least two lines of prior therapy [25]. However, to date all studies had been performed in patients with recurrent disease and the SOLO1 study was the first to evaluate the role of PARP inhibition in the first line setting when treatment has curative potential.

\section{First line therapy in advanced ovarian cancer}

The SOLO1 trial should be considered a landmark study. It the first trial to demonstrate meaningful PFS improvement in the first line setting for patients with advanced ovarian cancer. Progression free survival can be viewed as an indicator of the effectiveness of first line therapy, with little improvement observed over the past two decades. Carboplatin and paclitaxel has been the standard of care since the introduction of paclitaxel in the 1990s. Progression free survival is affected by the amount of residual disease, quality of cytoreductive surgery and BRCA status [26-28]. Maintenance chemotherapy, high-dose chemotherapy and weekly dose-scheduling have not reduced recurrence rates $[29,30]$. The addition of bevacizumab to chemotherapy followed by maintenance bevacizumab improves PFS by around four months, with the greatest benefit observed with more advanced disease [31,32]. However, a recent analysis of the GOG- 
218 data, suggested that $B R C A 1 / 2$ mutant patients (and those with other homologous recombination repair mutations) did not confer a statistically significant prolongation in PFS with extended bevacizumab use [28]. Whilst BRCA1/2 mutation carriers with advanced disease have superior PFS and OS compared to $B R C A 1 / 2$ wild-type patients $[28,33,34]$, relapses still occur in the majority of patients within three years of diagnosis, with a high rate of first line therapy failure.

Within the SOLO1 trial, the placebo arm had a median PFS of 13.8 months. This was measured from the completion of chemotherapy, with randomisation approximately 6.5 months from diagnosis, giving a median PFS from diagnosis of around 20.3 months. This is consistent with results reported in studies of carboplatin and paclitaxel in patients with newly diagnosed ovarian cancer and a BRCA1/2 mutation [28,35]. Whilst the median PFS has not yet been reached for the olaparib arm, a sensitivity analysis of investigator-assessed PFS was performed to evaluate for possible attrition bias. This demonstrated a median PFS of approximately 36 months more in the olaparib group than in the placebo group. Furthermore, the KaplanMeier estimate of the rate of freedom from disease progression and from death at four years was $53 \%$ with olaparib compared to $11 \%$ with placebo. This clinically meaningful prolongation in PFS was observed despite the median duration of olaparib treatment of 24.6 months. Many patients in this study were able to stop trial intervention at two years and live progression free for several months without treatment. In keeping with this, the median time to the first subsequent therapy or death was 51.8 months in the olaparib group and 15.1 months in the placebo group (HR $0.30 ; 95 \% \mathrm{Cl}, 0.22$ to 0.40$)$ [8].

Patients with newly diagnosed ovarian cancer are the only patients with ovarian cancer treated with curative intent. Whilst the OS data from SOLO1 are immature, the fact that more than half of the patients treated with olaparib were free from progression at four years raises the very real potential that this intervention may result in long-term durable benefit or even cure in a sub-set of patients.

\section{Patient population in SOLO1 - Does it compare to clinical practice?}

The patient population of SOLO1 was well matched, as expected, between the two groups. The majority had no evidence of disease following chemotherapy (82\%), were ECOG performance status zero (78\%) and had a CA-125 level within the normal range (95\%). Whilst this population is of better prognosis than that observed in everyday clinical practice, benefits for olaparib were observed in all subgroups including those patients with features which predict poorer prognosis including those with residual disease (HR 0.44; 95\% Cl 0.25-0.77), stage IV disease (HR 0.49; 95\% Cl 0.25-0.94), and ECOG performance status 1 (HR $0.38 ; 95 \% \mathrm{Cl} 0.21-0.68$ ) [8]. The data from the sub-group analysis supports the use of olaparib in all subgroups and suggests that this will translate into clinical benefit for all patients, including those with clinical features more typical for the non-trial patient population. 


\section{Olaparib maintenance in BRCA1/2 mutant ovarian cancer: first or second line?}

The impressive results from SOLO1 follow on from those reported in SOLO2 [6] Here, patients with advanced recurrent high-grade ovarian cancer and a BRCA1/2 mutation were randomised to olaparib or placebo maintenance following a response to platinum-based chemotherapy with a significant improvement in PFS observed with olaparib; 19.1 months versus 5.5 months on placebo (HR 0.30; 95\% $\mathrm{Cl} 0.22-0.41)$ [6]. This raises the question as to whether patients with $B R C A 1 / 2$ mutant ovarian cancer should be treated with olaparib maintenance in the first line or relapsed setting. Within SOLO2, 58\% of patients entered after second line platinum chemotherapy. The median PFS with olaparib was 19.1 months, with $43 \%$ of patients free from progression at 24 months [6]. In comparison $74 \%$ of patients on olaparib were free from progression at two-years within SOLO1. Furthermore, the absolute longer PFS with olaparib than with placebo seen in the sensitivity analysis in SOLO1 is substantially greater than that seen with SOLO2 (and with other PARP inhibitors in relapsed disease trials) [2,5,6]. Together these results suggest that olaparib maintenance in $B R C A 1 / 2$ patients has the greatest benefit in the first line setting. Furthermore, some patients (e.g. those who develop platinum resistance) are not eligible to receive olaparib as a second-line therapy and therefore the earlier introduction of these drugs will offer the opportunity for a greater number of patients to benefit and may even delay the onset of platinum resistance in this group.

There was a theoretical concern that treatment with PARP inhibitors may reduce response rate and duration of response to subsequent lines of therapies. This has not been borne out in the PARP maintenance studies performed in the relapsed setting, as evident by the prolonged time to second disease progression observed with both olaparib and niraparib compared to placebo $[5,6]$. In keeping with this, there was improvement in all efficacy endpoints with olaparib in SOLO1 (Table 1) suggesting that olaparib does not reduce the patients' ability to benefit from subsequent therapy. A significant increase in second progression free survival was observed with olaparib (Kaplan-Meier estimate of the rate of freedom from second disease progression or death at 3 years $75 \%$ with olaparib versus $60 \%$ with placebo; $\mathrm{HR} 0.50 ; 95 \% \mathrm{Cl}$, 0.35 to $0.72 ; p<0.001)$. The median second PFS was 41.9 months in the placebo group, this may be partially explained by the observation that $35 \%$ of these patients subsequently received treatment with a PARP inhibitor.

\section{Late toxicity}

An additional concern with early PARP inhibitor use is whether long-term use of agents that interfere with repair of double-stranded DNA breaks result in the development of the myelodysplastic syndrome (MDS) and acute leukaemia (AML). Within SOLO1 the incidence of AML reported was $1 \%$ in patients who received olaparib, which is consistent with the incidence of the MDS or AML reported in the SOLO2 trial (2\%) and 
other trials of PARP inhibitors [2-6]. To date, these data do not support the concern that earlier introduction of PARP inhibitors is detrimental but longer-term follow-up is essential to answer this question.

\section{Conclusions}

The significant difference in PFS seen in SOLO1, with the median PFS not yet reached and the estimation from the PFS2 results suggesting that it is likely to be longer 48 months provides hope that for these women there will be long-term remission and even possible cure. This group of women includes up to $22 \%$ of patients with high grade ovarian cancer, harbouring either a germline or somatic mutation in BRCA1/2. Whilst the OS data are not mature, the impressive data seen to date suggests that olaparib should be included in daily practice as maintenance therapy in this patient population. Interestingly, this is the first trial with a PARP inhibitor where a planned discontinuation of drug occurred after 24 months in patients without disease progression.

Ongoing and future trials will explore the role of PARP inhibition in patients regardless of $B R C A$ status. This includes GOG 3005, which is examining the concomitant and maintenance use of veliparib in the primary setting in patients with high-grade serous ovarian cancer (ClinicalTrials.gov identifier: NCT02470585) and the PRIMA study (NCT02655016) comparing maintenance niraparib or placebo therapy in patients with suboptimal stage III or stage IV disease following surgery and chemotherapy. The PAOLA-1 study is evaluating the addition of olaparib or placebo maintenance to bevacizumab following first-line therapy (NCT02477644). This study is testing the hypothesis that bevacizumab increases the activity of PARP inhibitor, possibly due to increasing the degree of homologous deficiency in the tumour [36]. A phase 2 study combing olaparib and the VEGF receptor inhibitor cediranib supports this approach with the greatest benefit observed in the BRCA wildtype group [37].

Finally, there is increasing interest in the combination of PARP inhibitors with checkpoint inhibitors such as anti-PD1/PDL1 and anti-CTLA4 antibodies. Single agent therapy with checkpoint inhibitors has demonstrated only modest activity in the treatment of recurrent ovarian cancer to date [38] and it is hypothesised that checkpoint inhibitor benefit is improved by combining with a PARP inhibitor. This rationale is based on two hypotheses; firstly, tumours with a BRCA1/2 mutation or an HRR defect have a higher mutational burden and therefore an elevated neo-antigen load, which is thought to elicit an increased antitumour immune response $[39,40]$. Secondly, PARP inhibitor treatment up regulates PD-L1 expression in vivo and in vitro [41] and in the absence of a functional BRCA pathway, there is activation of the innate immune response via the STING/TKB1/IRF3 response [42], which might influence the antitumor effect of a PARP inhibitor and checkpoint inhibitor combination. A number of phase 3 trials combing PARP inhibitor with checkpoint inhibition in the first line setting are underway. This includes; ATHENA (NCT03522246), combining rucaparib with nivolumab in the maintenance setting, DUO-O (NCT03737643) concomitant 
durvalumab and bevacizumab with chemotherapy followed by durvalumab, bevacizumab and olaparib maintenance and FIRST (NCT03602859) concomitant TSR-042 (anti-PD1 antibody) with chemotherapy followed by maintenance TSR-042 and niraparib. However, these trials were designed before the results of SOLO1 appeared and although there is stratification for the presence of a BRCA mutation, an alteration of the design may be required, so that patients with a $B R C A$ mutation do not miss the opportunity of receiving first-line maintenance olaparib.

Whilst it remains to be seen whether the dramatic improvement in PFS translate to an overall survival benefit or even an improved cure rate, the results from SOLO1 strongly supports the use of olaparib maintenance therapy in patients with $B R C A$ mutant ovarian cancer. It is therefore imperative that BRCA testing is performed in all patients with advanced ovarian cancer in a timely manner to allow them to benefit from this therapy, if appropriate. Ongoing studies will establish whether a similar benefit for first-line maintenance PARP inhibitor treatment is seen in BRCA1/2 wild-type patients and whether the response can be augmented with combination therapy.

\section{Executive Summary}

\section{Advanced ovarian cancer}

- Despite curative intent, combined-modality treatment with surgery and platinum-based chemotherapy has poor cure rates, with 5 -year survival ranging from $28 \%$ for FIGO stage 3 to $19 \%$ with FIGO stage 4.

- Approximately $20 \%$ of all high grade ovarian cancers harbour a germline or somatic $B R C A 1 / 2$ mutation

\section{First-line therapy for advanced ovarian cancer}

- Limited improvement in first-line PFS over two decades since the introduction of carboplatin and paclitaxel.

- The majority of patients with advanced disease will recur within three years of diagnosis.

\section{SOLO1 trial design}

- Patients with advanced high-grade serous or endometrioid ovarian cancer with a mutation in $B R C A 1 / 2$, and who had a complete or partial clinical response after platinum-based chemotherapy, randomly assigned, in a 2:1 ratio, to receive olaparib tablets (300 mg twice daily, $n=260$ ) or placebo $(n=131)$.

- Trial intervention was continued until disease progression. Patients who had no evidence of disease at two years discontinued treatment, but patients who had a partial response at two years continue receiving treatment. The primary end point was investigator assessed progression-free

\section{Results} survival.

- After median follow-up of 41 months, the risk of investigator assessed disease progression or death (data maturity $51 \%$ ) was $70 \%$ lower with olaparib than with placebo (60\% versus $27 \%$; HR 0.30 ; $95 \% \mathrm{Cl}, 0.23$ to $0.41, \mathrm{p}<0.001$ )

- PFS (as assessed by BICR, data maturity, 38\%), estimated rate of freedom from disease progression/ death at 3 years $69 \%$ olaparib versus $35 \%$ placebo (HR $0.28 ; 95 \% \mathrm{Cl}, 0.20$ to 0.39 , $\mathrm{p}<0.001)$.

- Adverse events were consistent with the known toxic effects of olaparib. Serious adverse events occurred in $21 \%$ of olaparib and $12 \%$ of placebo treated patients. AML occurred in $1 \%$ of the olaparib group and in none in the placebo group, new primary cancers occurred in $2 \%$ in both groups.

Impact and implications 
- Olaparib maintenance should become the new standard of care for women with BRCA1/2 mutant, advanced ovarian cancer following a response to first line platinum-based chemotherapy

- Early testing for BRCA status is required

Unanswered questions

- Will the dramatic improvement in progression-free survival translate to an overall survival benefit or even an improved cure rate?

- Is there a similar benefit for first-line maintenance PARP inhibitor treatment in patients whose tumours are BRCA1/2 wild-type?

- Does long-term use of PARP inhibitors that interfere with repair of double-stranded DNA breaks result in the development of the myelodysplastic syndrome and acute leukemia? To date, across published studies, accumulating evidence suggests that the risk is fairly low $(<2 \%)$, but longer-term follow-up is essential. 


\section{Figure and Tables}

\section{Figure 1: SOLO1 trial design}

FIGO, International Federation of Gynecology and Obstetrics; ECOG, Eastern Cooperative Oncology Group; RECIST, Response Evaluation Criteria in Solid Tumours; PFS, Progression Free Survival; BICR, blinded independent central review; PFS2, time to second progression or death; HRQoL, health-related quality of life; FACT-O, Functional Assessment of Cancer Therapy - Ovarian Cancer; TOI, Trial Outcome Index

- Newly diagnosed, FIGO stage III-IV, high-grade serous or endometrioid ovarian, primary peritoneal or fallopian tube cancer

- Upfront or interval attempt at optimal cytoreductive surgery for stage III disease and either biopsy and/or upfront or interval cytoreductive surgery for stage IV disease

- Germline or somatic $B R C A \mathrm{~m}$

- ECOG performance status $0-1$

- In clinical complete response or partial response after platinum-based chemotherapy

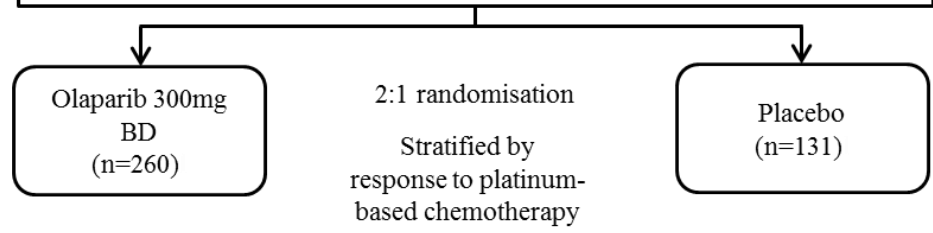

- Study treatment continued until disease progression

- Patients with no evidence of disease at 2 years stopped treatment

- Patients with a partial response at 2 years could continue treatment

PRIMARY ENDPOINT

Investigator-assessed PFS (modified RECIST 1.1)

SECONDARY ENDPOINTS

- PFS using BICR

- PFS2

- Overall survival

- Time from randomization to first subsequent therapy or death

- Time from randomization to second subsequent therapy or death

- HRQoL (FACT-O TOI score)

\begin{tabular}{|l|c|c|c|}
\hline Efficacy End Points & $\begin{array}{l}\text { Olaparib } \\
\mathbf{N = 2 6 0}\end{array}$ & $\begin{array}{l}\text { Placebo } \\
\mathbf{N = 1 3 1}\end{array}$ & $\begin{array}{l}\text { Hazard Ratio } \\
\mathbf{9 5 \%} \mathbf{~ C l}\end{array}$ \\
\hline Median PFS* (months) & $\begin{array}{c}\text { Not } \\
\text { reached }\end{array}$ & 13.8 & $\begin{array}{c}0.30 \\
(0.23-0.41)\end{array}$ \\
\hline $\begin{array}{l}\text { Median time to first subsequent } \\
\text { therapy or death (months) }\end{array}$ & 51.8 & 15.1 & $\begin{array}{c}0.30 \\
(0.22-0.40)\end{array}$ \\
\hline Median PFS2 (months) & $\begin{array}{c}\text { Not } \\
\text { reached }\end{array}$ & 41.9 & $\begin{array}{c}0.50 \\
(0.35-0.72)\end{array}$ \\
\hline $\begin{array}{l}\text { Median time to second subsequent } \\
\text { therapy or death (months) }\end{array}$ & $\begin{array}{c}\text { Not } \\
\text { reached }\end{array}$ & 40.7 & $\begin{array}{c}0.45 \\
(0.32-0.63)\end{array}$ \\
\hline
\end{tabular}

Table 1: Summary of efficacy end points. * investigator assessed, PFS2, median second Progression Free Survival- time to second progression or death, $\mathrm{Cl}$ - confidence interval. 


\section{References}

1. Jayson GC, Kohn EC, Kitchener HC, Ledermann JA. Ovarian cancer. Lancet, 384(9951), 13761388 (2014).

2. Coleman RL, Oza AM, Lorusso D et al. Rucaparib maintenance treatment for recurrent ovarian carcinoma after response to platinum therapy (ARIEL3): a randomised, double-blind, placebocontrolled, phase 3 trial. Lancet, 390(10106), 1949-1961 (2017).

3. Ledermann J, Harter P, Gourley $\mathrm{C}$ et al. Olaparib maintenance therapy in platinum-sensitive relapsed ovarian cancer. The New England Journal of Medicine, 366(15), 1382-1392 (2012).

${ }^{* *}$ First trial demonstrating PFS benefit of maintenance olaparib

4. Ledermann J, Harter P, Gourley C et al. Olaparib maintenance therapy in patients with platinumsensitive relapsed serous ovarian cancer: a preplanned retrospective analysis of outcomes by BRCA status in a randomised phase 2 trial. The Lancet Oncology, 15(8), 852-861 (2014).

5. Mirza MR, Monk BJ, Herrstedt J et al. Niraparib Maintenance Therapy in Platinum-Sensitive, Recurrent Ovarian Cancer. The New England Journal of Medicine, (2016).

6. Pujade-Lauraine E, Ledermann JA, Selle $F$ et al. Olaparib tablets as maintenance therapy in patients with platinum-sensitive, relapsed ovarian cancer and a BRCA1/2 mutation (SOLO2/ENGOT-Ov21): a double-blind, randomised, placebo-controlled, phase 3 trial. The Lancet Oncology, 18(9), 1274-1284 (2017).

*Olaparib maintenance therapy in the second line setting

7. Friedlander M, Matulonis U, Gourley $\mathrm{C}$ et al. Long-term efficacy, tolerability and overall survival in patients with platinum-sensitive, recurrent high-grade serous ovarian cancer treated with maintenance olaparib capsules following response to chemotherapy. Br J Cancer, 119(9), 10751085 (2018).

8. Moore K, Colombo N, Scambia G et al. Maintenance Olaparib in Patients with Newly Diagnosed Advanced Ovarian Cancer. The New England Journal of Medicine, 379(26), 2495-2505 (2018).

9. Prakash R, Zhang Y, Feng W, Jasin M. Homologous recombination and human health: the roles of BRCA1, BRCA2, and associated proteins. Cold Spring Harbor Perspectives in Biology, 7(4), a016600 (2015).

10. Kanchi KL, Johnson KJ, Lu C et al. Integrated analysis of germline and somatic variants in ovarian cancer. Nature Communications, 5, 3156 (2014).

11. Cancer Genome Atlas Research N. Integrated genomic analyses of ovarian carcinoma. Nature, 474(7353), 609-615 (2011).

12. Bryant HE, Schultz N, Thomas HD et al. Specific killing of BRCA2-deficient tumours with inhibitors of poly(ADP-ribose) polymerase. Nature, 434(7035), 913-917 (2005).

* Key paper showing BRCA/PARP synthetic lethality

13. Farmer $\mathrm{H}, \mathrm{McCabe} \mathrm{N}$, Lord $\mathrm{CJ}$ et al. Targeting the DNA repair defect in BRCA mutant cells as a therapeutic strategy. Nature, 434(7035), 917-921 (2005).

* Key paper showing BRCA/PARP synthetic lethality

14. O'Connor MJ. Targeting the DNA Damage Response in Cancer. Mol Cell, 60(4), 547-560 (2015).

15. Bell $D$, Berchuck $A$, Birrer $M$ et al. Integrated genomic analyses of ovarian carcinoma. Nature, 474(7353), 609-615 (2011).

16. Pennington KP, Walsh $\mathrm{T}$, Harrell $\mathrm{Ml}$ et al. Germline and somatic mutations in homologous recombination genes predict platinum response and survival in ovarian, fallopian tube, and peritoneal carcinomas. Clinical Cancer Research,, 20(3), 764-775 (2014).

17. EMA. 2018 [EMA approval of olaparib]. Available from: http://www.ema.europa.eu/ema/index.jsp?curl=pages/medicines/human/medicines/003726/human med 001831.jsp\&mid=WC0b01ac058001d124.

$\begin{array}{lllllll} & & & \\ 18 . & E M A & 2017 & \text { [EMA } & \text { approval of } & \end{array}$ https://www.ema.europa.eu/en/medicines/human/EPAR/zejula.

19. EMA. 2018 [EMA approval of rucaparib ]. Available from: https://www.ema.europa.eu/en/medicines/human/summaries-opinion/rubraca.

20. FDA U. FDA approval of niraparib 2017 [Available from:

https://www.fda.gov/Drugs/InformationOnDrugs/ApprovedDrugs/ucm548487.htm. 
21. FDA U. FDA approval of olaprib 2017 [Available from: https://www.fda.gov/drugs/informationondrugs/approveddrugs/ucm572143.htm.

22. FDA U. FDA approval of rucaparib 2018 [cited 2019. Available from: https://www.fda.gov/Drugs/InformationOnDrugs/ApprovedDrugs/ucm533891.htm.

23. Gelmon KA, Tischkowitz M, Mackay $\mathrm{H}$ et al. Olaparib in patients with recurrent high-grade serous or poorly differentiated ovarian carcinoma or triple-negative breast cancer: a phase 2, multicentre, open-label, non-randomised study. The Lancet Oncology, 12(9), 852-861 (2011).

24. Kaufman B, Shapira-Frommer R, Schmutzler RK et al. Olaparib Monotherapy in Patients With Advanced Cancer and a Germline BRCA1/2 Mutation. Journal of Clinical Oncology,, 33(3), 244250 (2015).

25. Oza AM, Tinker AV, Oaknin A et al. Antitumor activity and safety of the PARP inhibitor rucaparib in patients with high-grade ovarian carcinoma and a germline or somatic BRCA1 or BRCA2 mutation: Integrated analysis of data from Study 10 and ARIEL2. Gynecologic Oncology, 147(2), 267-275 (2017).

26. Polterauer S, Vergote I, Concin $\mathrm{N}$ et al. Prognostic value of residual tumor size in patients with epithelial ovarian cancer FIGO stages IIA-IV: analysis of the OVCAD data. International Journal of Gynecological Cancer,, 22(3), 380-385 (2012).

27. Harter $\mathrm{P}$, Muallem ZM, Buhrmann $\mathrm{C}$ et al. Impact of a structured quality management program on surgical outcome in primary advanced ovarian cancer. Gynecologic Oncology, 121(3), 615-619 (2011).

28. Norquist BM, Brady MF, Harrell MI et al. Mutations in Homologous Recombination Genes and Outcomes in Ovarian Carcinoma Patients in GOG 218: An NRG Oncology/Gynecologic Oncology Group Study. Clinical Cancer Research, 24(4), 777-783 (2018).

29. Clamp A MI, Dean A, Gallardo D, Kim JW, O'Donnell DM, Hook J,, Coyle C BS, Brenton J, Naik R, PerrenT, Sundar S, Cook L,, James E SA, Stenning S, Kaplan R, Ledermann J. ICON8: A GGIG Phase III Randomised Trial Evaluating Weekly Dose-Dense Chemotherapy Integration In FirstLine Epithelial Ovarian/Fallopian Tube/Primary Peritoneal Carcinoma (EOC) Treatment: Results Of Primary Progression Free Survival (PFS) Analysis Annals of Oncology, 28(viii332-viii358) (2017).

30. Markman M, Liu PY, Wilczynski S et al. Phase III randomized trial of 12 versus 3 months of maintenance paclitaxel in patients with advanced ovarian cancer after complete response to platinum and paclitaxel-based chemotherapy: a Southwest Oncology Group and Gynecologic Oncology Group trial. Journal of Clinical Oncology,, 21(13), 2460-2465 (2003).

31. Burger RA, Brady MF, Bookman MA et al. Incorporation of bevacizumab in the primary treatment of ovarian cancer. The New England Journal of Medicine, 365(26), 2473-2483 (2011).

32. Oza AM, Cook AD, Pfisterer $\mathrm{J}$ et al. Standard chemotherapy with or without bevacizumab for women with newly diagnosed ovarian cancer (ICON7): overall survival results of a phase 3 randomised trial. The Lancet Oncology, 16(8), 928-936 (2015).

33. Alsop K, Fereday S, Meldrum C et al. BRCA mutation frequency and patterns of treatment response in BRCA mutation-positive women with ovarian cancer: a report from the Australian Ovarian Cancer Study Group. Journal of Clinical Oncology,, 30(21), 2654-2663 (2012).

34. Cass I, Baldwin RL, Varkey T, Moslehi R, Narod SA, Karlan BY. Improved survival in women with BRCA-associated ovarian carcinoma. Cancer, 97(9), 2187-2195 (2003).

35. Alsop K, Fereday S, Meldrum $\mathrm{C}$ et al. BRCA Mutation Frequency and Patterns of Treatment Response in BRCA Mutation-Positive Women With Ovarian Cancer: A Report From the Australian Ovarian Cancer Study Group. Journal of Clinical Oncology,, 30(21), 2654-2663 (2012).

36. Dean E, Middleton MR, Pwint T et al. Phase I study to assess the safety and tolerability of olaparib in combination with bevacizumab in patients with advanced solid tumours. Br J Cancer, 106(3), 468-474 (2012).

37. Liu JF, Barry WT, Birrer M et al. Combination cediranib and olaparib versus olaparib alone for women with recurrent platinum-sensitive ovarian cancer: a randomised phase 2 study. The Lancet Oncology, (2014).

38. Matulonis UA S-FR, Santin A, Lisyanskaya AS, Pignata S, Vergote I, Raspagliesi F, Sonke GS, Michael Birrer M, Provencher DM, Sehouli J, Nicoletta Colombo N, González-Martín A, Oaknin A, Ottevanger PB, Rudaitis V, Katchar K, Wang Z, Ruman J, Ledermann JA. Antitumor activity and 
safety of pembrolizumab in patients with advanced recurrent ovarian cancer: Interim results from the phase 2 KEYNOTE-100 study. Journal of Clinical Oncology 15 (2018).

39. Higuchi T, Flies DB, Marjon NA et al. CTLA-4 Blockade Synergizes Therapeutically with PARP Inhibition in BRCA1-Deficient Ovarian Cancer. Cancer Immunology Research, (2015).

40. Huang J, Wang L, Cong $Z$ et al. The PARP1 inhibitor BMN 673 exhibits immunoregulatory effects in a Brca1(-/-) murine model of ovarian cancer. Biochem Biophys Res Commun, 463(4), 551-556 (2015).

41. Jiao S, Xia W, Yamaguchi $\mathrm{H}$ et al. PARP Inhibitor Upregulates PD-L1 Expression and Enhances Cancer-Associated Immunosuppression. Clinical Cancer Research, (2017).

* Scientific rationale for PARP and anti PD-L1 therapy combinations

42. Parkes EE, Walker SM, Taggart LE et al. Activation of STING-Dependent Innate Immune Signaling By S-Phase-Specific DNA Damage in Breast Cancer. Journal of the National Cancer Institute, 109(1) (2017). 